\title{
SUMS AND PRODUCTS OF CONTINUED FRACTIONS
}

\author{
T. W. CUSICK
}

ABstract. It is proved that every real number is representable as a sum of two real numbers each of which has a fractional part whose continued fraction expansion contains no partial quotient less than 2, and that every real number not less than one is representable as a product of two real numbers with the same property.

Marshall Hall, Jr. [1] proved that every real number is representable as a sum of two real numbers each of which has a fractional part whose continued fraction expansion contains no partial quotient greater than 4 , and that every real number not less than 1 is representable as a product of two real numbers with the same property. In this paper I prove the following related results (here it is convenient to regard 0 as the reciprocal of an infinite partial quotient):

THEOREM 1. Every real number is representable as a sum of two real numbers each of which has a fractional part whose continued fraction expansion contains no partial quotient less than 2.

THEOREM 2. Every real number not less than 1 is representable as a product of two real numbers each of which has a fractional part whose continued fraction expansion contains no partial quotient less than 2.

The proofs of both theorems use ideas of Hall [1, pp. 967-970] on Cantor point sets, defined as follows: Take a closed interval $A=[x, x+a]$ on the real line, and remove from it a middle open interval $A_{12}=\left(x+a_{1}, x+a_{1}+a_{12}\right)$; two closed intervals $A_{1}=\left[x, x+a_{1}\right]$ and $A_{2}=\left[x+a_{1}+a_{12}, x+a\right]$ remain. If both $A_{1}$ and $A_{2}$ are subdivided in the same way and the process is continued with every closed interval which arises at each stage, then the points of $A$ which are not in any of the deleted open intervals form a Cantor point set, denoted by $C(A)$.

I first show that for each integer $k \geqq 2$, the set $S(k)$ of numbers $\alpha$ such that $0 \leqq \alpha \leqq k^{-1}$ and $\alpha$ has a continued fraction containing no partial quotient less than $k$, is a Cantor point set in the interval $A=\left[0, k^{-1}\right]$.

Let $C F\left(a_{0}, a_{1}, a_{2}, \cdots\right)$ denote the continued fraction with partial

Received by the editors February 24, 1970.

AMS 1969 subject classifications. Primary 1031; Secondary 0415.

Key words and phrases. Continued fractions, Cantor sets, sums of sets. 
quotients $a_{0}, a_{1}, a_{2}, \cdots$. In defining the subdivisions of $A$ which produce $S(k)$, two types of intervals with rational endpoints need to be considered. Intervals of the first type have the form

$$
\left[C F\left(0, a_{1}, \cdots, a_{n}\right), C F\left(0, a_{1}, \cdots, a_{n+1}\right)\right]
$$

where $n$ is an even integer and $a_{i} \geqq k(i=1,2, \ldots)$; intervals of the second type have the form

$$
\left[C F\left(0, a_{1}, \cdots, a_{n}\right), C F\left(0, a_{1}, \cdots, a_{n-1}\right)\right]
$$

where $n$ is an even integer and $a_{i} \geqq k(i=1,2, \cdots)$. Thus $A$ is an interval of the first type with $n=0$.

In the subdivision process, the interval

$$
\left(C F\left(0, a_{1}, \cdots, a_{n}, a_{n+1}+1\right), C F\left(0, a_{1}, \cdots, a_{n+1}, k\right)\right)
$$

is removed from each interval (1) of first type and the interval

$$
\left(C F\left(0, a_{1}, \cdots, a_{n}, k\right), C F\left(0, a_{1}, \cdots, a_{n-1}, a_{n}+1\right)\right)
$$

is removed from each interval (2) of second type. In both cases the removal of the middle interval leaves behind an interval of first type on the left side and an interval of second type on the right side. Therefore the subdivision process can be continued, and the resulting Cantor point set is clearly $S(k)$ since the excluded intervals (3), (4) contain no elements of $S(k)$ and every number not in $S(k)$ is in some excluded interval.

Define the sum $U+V$ of two point sets $U$ and $V$ to be the set of all $u+v$, where $u$ is in $U$ and $v$ is in $V$. Let $C(A)$ and $C(B)$ be two Cantor point sets in the intervals $A$ and $B$. Hall $[1$, p. 968] proved the following lemma, which gives a sufficient (but not necessary) condition for $C(A)+C(B)$ to cover the whole interval $A+B$.

Lemma 1. Suppose that the ratio of the length of the longer of the two intervals $A, B$ to the length of the shorter does not exceed 3. Suppose further that in the subdivision processes which define $C(A)$ and $C(B)$, at each stage of the subdivision the middle interval removed from each subinterval is not longer than either subinterval retained. Then $C(A)$ $+C(B)$ covers the whole interval $A+B$.

Theorem 1 is proved by using Lemma 1 to show that $S(2)+S(2)$ $=[0,1]$. Let $p_{j} / q_{j}$ denote $C F\left(0, a_{1}, \cdots, a_{j}\right)$ in lowest terms. A simple calculation shows that the length of the interval (1) of first type is $\left(q_{n} q_{n+1}\right)^{-1}$ and that the ratios of the length of the excluded interval (3) to the lengths of the retained intervals on the left and right, respectively, are 


$$
(k-1) q_{n} /\left(k q_{n+1}+q_{n}\right) \text { and }(k-1) q_{n+1} /\left(q_{n+1}+q_{n}\right) .
$$

Similarly, the length of the interval (2) of second type is $\left(q_{n-1} q_{n}\right)^{-1}$ and the ratios of the length of the excluded interval (4) to the lengths of the retained intervals on the left and right, respectively, are

$$
(k-1) q_{n} /\left(q_{n}+q_{n-1}\right) \text { and }(k-1) q_{n-1} /\left(k q_{n}+q_{n-1}\right) .
$$

Thus Lemma 1 applies to $S(k)+S(k)$ for $k=2$ (but not for $k \geqq 3$ ) and Theorem 1 is proved.

Let $I_{n}$ denote the interval $\left[n, n+\frac{1}{2}\right](n=0,1,2, \cdots)$, and consider the sequence of pairs of intervals $I_{1}, I_{1} ; I_{1}, I_{2} ; I_{1}, I_{3} ; \ldots$; $I_{n}, I_{n} ; I_{n}, I_{n+1} ; I_{n}, I_{n+2} ; \cdots(n=1,2, \cdots)$. It is easy to verify that every $x \geqq 1$ is representable as $\alpha \beta$, where $\alpha$ is in the first interval of some pair in the sequence and $\beta$ is in the second interval of the same pair. Let $C\left(I_{n}\right)$ denote the Cantor point set formed by adding $n$ to each element of $S(2)$; let $I_{n}^{*}$ denote the interval $\left[\log n, \log \left(n+\frac{1}{2}\right)\right]$ and let $C\left(I_{n}^{*}\right)$ denote the Cantor point set formed by taking the logarithm of each element of $C\left(I_{n}\right)$. As in [1, pp. 974-975], Theorem 2 is proved if $C\left(I_{n}^{*}\right)+C\left(I_{n+j}^{*}\right)=I_{n}^{*}+I_{n+j}^{*}$ for each $n=1,2, \cdots$ and each $j=0,1$ or 2 . Hence Theorem 2 is proved if the hypotheses of Lemma 1 are satisfied for $A=I_{n}^{*}, B=I_{n+j}^{*}$.

The first supposition in Lemma 1 is easily verified by comparing the length of $I_{n}^{*}$ with the lengths of the shorter intervals $I_{n+1}^{*}$ and $I_{n+2}^{*}$. The second supposition in Lemma 1 can be verified by using the following lemma, which is a modified version of a lemma of Hall $[1$, p. 975$]$.

Lemma 2. Let $A=[a, b]$ be any interval of positive numbers, and let $A$ be subdivided into three intervals $A_{1}=[a, a+r], A_{12}=[a+r, a+r+s]$, $A_{2}=[a+r+s, a+r+s+t]$, where $a+r+s+t=b$. Let $A^{*}=[\log a$, $\log b]$ and let $A_{1}^{*}, A_{12}^{*}, A_{2}^{*}$ denote the intervals in the corresponding subdivision of $A^{*}$. Then $A_{12}^{*}$ is not longer than either of $A_{1}^{*}$ and $A_{2}^{*}$ if $b(t-s) \geqq t^{2}$.

If in Lemma $2 A$ is taken to be any of the intervals (1) or (2) which arise in the subdivision process which produces $C\left(I_{m}\right)(m \geqq 1)$, then $b>1$. Calculation shows that for any such interval, the expression corresponding to $t^{-2}(t-s)$ exceeds either $q_{n} q_{n+1} \geqq 2$ (if the interval is of the first type )or $q_{n-1} q_{n} \geqq 10$ (if the interval is of the second type, which can happen only for $n \geqq 2$ in (2)). This completes the proof of Theorem 2.

I conclude with some elementary remarks about Theorem 1 . Since $S(k)+S(k)$ is contained in $\left[0,2 k^{-1}\right]$, which cannot cover $[0,1]$ for 
$k>2$, it is trivial that Theorem 1 becomes false if the lower bound 2 for the partial quotients is replaced by any larger integer. Even the equality $S(k)+S(k)=\left[0,2 k^{-1}\right]$ is easily seen to be false for $k>2$, as consideration of the effect of the removal of the first middle interval in the subdivision process which defines $S(k)$ immediately shows. The question of determining the measure of the set $S(k)+S(k)$ is an interesting one. I conjecture that the measure is zero for $k>2$.

For any integer $m \geqq 3$, it is false that every real number is representable as a sum of two real numbers each of which has a fractional part whose continued fraction expansion contains no partial quotient less than 2 or greater than $m$; for it is clear that given any $m$, all real numbers with sufficiently small fractional part will not be representable. Thus Theorem 1 is best possible in a certain sense.

\section{REFERENCES}

1. Marshall Hall, Jr., On the sum and product of continued fractions, Ann. of Math. (2) 48 (1947), 966-993. MR 9, 226.

State University of New York at Buffalo, Amberst, New York 14226 